\title{
Enriching the annotation of Mycobacterium tuberculosis H37Rv proteome using remote homology detection approaches: Insights into structure and function
}

\author{
Gayatri Ramakrishnan ${ }^{1,2}$, Bernardo Ochoa-Montano ${ }^{4}$, Upadhyayula S. Raghavender ${ }^{5}$, Richa \\ Mudgal $^{1,2}$, Adwait G. Joshi ${ }^{5}$, Nagasuma R. Chandra ${ }^{3}$, Ramanathan Sowdhamini ${ }^{5}$, Tom L. Blundell ${ }^{4}$ \\ and Narayanaswamy Srinivasan ${ }^{2 \ddagger}$ \\ ${ }^{1}$ Indian Institute of Science Mathematics Initiative, ${ }^{2}$ Molecular Biophysics Unit, ${ }^{3}$ Department of \\ Biochemistry, Indian Institute of Science, Bangalore 560012, India \\ ${ }^{4}$ Department of Biochemistry, University of Cambridge, Cambridge CB1 2GA, UK \\ ${ }^{5}$ National Centre for Biological Sciences (Tata Institute of Fundamental Research), Gandhi Krishi Vignyan \\ Kendra Campus, Bangalore 560065, India \\ E-mail addresses:
}

Gayatri Ramakrishnan: rgayatri@mbu.iisc.ernet.in

Bernardo Ochoa-Montano: bernardo@cryst.bioc.cam.ac.uk

Upadhyayula S. Raghavender: upadhyayulas@ncbs.res.in

Richa Mudgal: rmudgal@mbu.iisc.ernet.in

Adwait G. Joshi: adwait@ncbs.res.in

Nagasuma R. Chandra: nchandra@biochem.iisc.ernet.in

Ramanathan Sowdhamini: mini@ncbs.res.in

Tom L. Blundell: tom@cryst.bioc.cam.ac.uk

${ }^{\ddagger}$ Corresponding author: Narayanaswamy Srinivasan

Email: ns@mbu.iisc.ernet.in

Phone: +91-80-2293-2837

Fax: +91-80-2360-0535

\section{Abbreviations}

(C) 2014. This manuscript version is made available under the Elsevier user license http://www.elsevier.com/open-access/userlicense/1.0/ 
ABC: ATP-binding cassette transporter family

AlignHUSH: Alignment of hidden Markov models using structural and hydrophobicity information

BLAST: Basic local alignment search tool

CATH: A hierarchical classification of domains in protein structures representing- class, architecture, topology and homologous superfamily

CopC: Copper resistance protein C family

DUFs: Domains of unknown function

ESAT-6-like: 6kDa secretory antigenic target

ESSTs: Environment specific substitution tables

GA: Gathering threshold

HMMs: Hidden Markov models

LAM: Lipoarabinomannan

LM: Lipomannan

mAGP: mycolyl-arabinogalactan-peptidoglycan

MatE: Multi-antimicrobial extrusion domain family

MFS: Major facilitator superfamily

MulPSSM: Multiple position-specific scoring matrices

NCBI: National centre for biotechnology information

OPT: Oligopeptide transporter protein family

PALI: Phylogeny and alignment of homologous protein structures

PEBP: Phosphatidyl ethanolamine-binding protein

PE: PE motif containing domain family

PPE: PPE motif containing domain family

PhdYeFM_antitox: Antitoxin Phd_YefM, type II toxin-antitoxin system 
PHYRE $^{2}$ : Protein homology recognition engine

PIN: PilT protein, N-terminal domain family

PP-binding: Phosphopantetheine attachment site

PSSMs: Position-specific scoring matrices

PTR: Proton-dependent oligopeptide transporter family

PURE: Prediction of unassigned regions

RPS-BLAST: Reverse position-specific iterative BLAST

SCOP: Structural classification of proteins

SUPFAM: A database of superfamily relationships

TctB: Tripartite tricarboxylate transporter family

UPFs: Uncharacterized protein families

WXG100: Protein of 100 residues with WXG

\section{Abstract}


The availability of the genome sequence of Mycobacterium tuberculosis H37Rv has encouraged determination of large numbers of protein structures and detailed definition of the biological information encoded therein; yet, the functions of many proteins in $M$. tuberculosis remain unknown. The emergence of multidrug resistant strains makes it a priority to exploit recent advances in homology recognition and structure prediction to re-analyse its gene products. Here we report the structural and functional characterization of gene products encoded in the M. tuberculosis genome, with the help of sensitive profile-based remote homology search and fold recognition algorithms resulting in an enhanced annotation of the proteome where $95 \%$ of the $M$. tuberculosis proteins were identified wholly or partly with information on structure or function. New information includes association of 244 proteins with 205 domain families and a separate set of new association of folds to 64 proteins. Extending structural information across uncharacterized protein families represented in the $M$. tuberculosis proteome, by determining superfamily relationships between families of known and unknown structures, has contributed to an enhancement in the knowledge of structural content. In retrospect, such superfamily relationships have facilitated recognition of probable structure and/or function for several uncharacterized protein families, eventually aiding recognition of probable functions for homologous proteins corresponding to such families. Gene products unique to mycobacteria for which no functions could be identified are 183. Of these 18 were determined to be $M$. tuberculosis specific. Such pathogen-specific proteins are speculated to harbour virulence factors required for pathogenesis. A re-annotated proteome of $M$. tuberculosis, with greater completeness of annotated proteins and domain assigned regions, provides a valuable basis for experimental endeavours designed to obtain a better understanding of pathogenesis and to accelerate the process of drug target discovery.

Keywords: Mycobacterium tuberculosis; Protein annotation; Remote homology detection; Sequence analysis

\section{Introduction}


Tuberculosis, a disease of antiquity, remains a global burden, reportedly being highest in Asia and Africa with an estimated death rate of 1.4 million per year (http://www.who.int/tb/). The availability of the genome sequence of Mycobacterium tuberculosis H37Rv [1] has played a major role in its functional characterization, but the emergence of multi-drug and extensively drug resistant strains necessitates further advances in understanding pathogenesis and its underlying complexity. Understanding physiological processes and differences at the molecular level has been a major endeavour for molecular biologists, and this has been addressed with the help of a myriad of experimental techniques and computational tools. At the computational level, the analysis of sequence information is no exception. Genome sequencing and related projects have led to a data deluge, with a growing disparity between available protein sequences and experimentally determined structures. About 104,000 experimentally determined structures of proteins are available in PDB (http://www.rcsb.org/) while the number of protein sequences deposited in public databases is over 35 million (http://www.ncbi.nlm.nih.gov/refseq/). Also, a considerable increase in the number of functionally uncharacterized proteins of known structure has been observed. Clearly, computational methods for protein function annotation and for investigation of evolutionary and structure-function relationships are becoming of increasing importance.

The complete genome of $M$. tuberculosis was sequenced and annotated over a decade ago with 3924 protein-coding genes [1] , followed by a subsequent re-annotation, which provided a revised version with 4006 protein-coding genes [2] . Functional annotation for $78 \%$ of these protein-coding genes was achieved using remote similarity detection tools, previously by our group [ㅁ]. A recent release of the genome (R27, March 2013) has resulted from an extensive manual curation [4], which now comprises 4018 protein-coding genes. Significant changes have been made to the genomic data and existing coding sequences. Many groups have made substantial contributions in an attempt to annotate the $M$. tuberculosis genome. Recent work on the structural proteome of $M$. tuberculosis [5] provides functional annotations for a large number of proteins as well as insights into predominant folds in the proteome. In a similar way, a genome-scale fold-recognition of the proteome, published recently []], provides considerable information on possible structures and functions of several un-annotated proteins. A novel crowd sourcing approach exercised to generate M. tuberculosis interactome for drug target identification has also proved successful in annotation of $87 \%$ of its gene products [7]. Since computational prediction of function is an on-going process based on ever growing information made available in public databases and the introduction of more powerful homology recognition techniques, a continuous refinement is thus essential to make the most of sequence data, ensuring its accuracy and relevance. 
Protein homology detection is a mainstay in the field of bioinformatics, playing a pivotal role in addressing issues concerning evolution of protein structures, functions and interactions. Many developments have been made in establishing evolutionary and structure-function relationships based on sequence information with the help of database similarity searches. The most widely used tool in identification of well-characterized homologues is BLAST []. However, reliable detection of homologues becomes skewed at very low sequence identities, i.e. <30\% [9] . Sensitive search procedures based on the use of profiles such as Position Specific Scoring Matrices (PSSMs) [10, 11], Environment Specific Substitution Tables (ESSTs) [12] and Hidden Markov Models (HMMs) [13] were then developed that are far more capable of detecting remote homologues with reasonable accuracy. To overcome the limitations posed by heuristics employed in speed and sensitivity of certain profile-based procedures, developments in the past decade include use of compositionally biased profiles [14], multiple profiles from alignments [15-18], intermediate sequence search [19, 20], sequence context-specific profiles [21] and profile-profile search procedures [22-24]. Such sensitive approaches can not only be used to mine sequence databases but structural databases as well. Since structural features are conserved better than the sequences in the course of evolution [25, 26], use of structural information to predict folds or biochemical motifs can very well provide useful insights in function prediction.

Inference of protein function is often made using assigned structural and functional domain families [27]. A domain family, represented as a statistical model such as an HMM profile, can capture information on sets of protein regions that share a significant degree of similarity obtained from multiple sequence alignment. Commonly used sequence and structure-based protein family resources include Pfam [28] , which provides comprehensive collection of sequence domains and SUPERFAMILY [29], which provides sequence profiles corresponding to structural classification of proteins by SCOP [잉. In our present study, we have used a combination of sensitive profile-based approaches in an attempt to achieve enriched annotation of the gene products encoded in $M$. tuberculosis genome, with the use of structural and functional domain families and in the process we have been able to predict functions for $\sim 89 \%$ of 4018 M. tuberculosis proteins. Such a combination of approaches has also been exploited in the past $[\underline{3}, \underline{31}, \underline{32}]$ for the analysis of genomic data. Sensitive approaches were also employed, coupled with manual intervention, to explore reliable detection of sequence-structure and sequence-function relationships for proteins for which no domains could be assigned. Such a protocol enriched annotation of the $M$. tuberculosis proteome by an additional 6\% (269 of 4018 proteins) totalling to $~ 95 \%$ of the proteome. The functional annotations for M. tuberculosis proteome can thus serve as guiding tools to understand the complexity of its pathogenesis and accelerate the process of identification of drug targets. 
Domain re-annotation of Mycobacterium tuberculosis proteome

2. Methodology 
Protein sequences of $M$. tuberculosis H37Rv genome were retrieved from TubercuList (http://tuberculist.epfl.ch/). The updated release of the proteome data as on March 2013 (sequences of 4018 gene products) has been used in the current analysis. This section has been categorized in to the following sub-sections:

i. Recognition of structural and functional domains: Each of the M. tuberculosis protein sequences is searched against sequence and structure-based protein family resources using a combination of sensitive profile-based techniques followed by critical assessment of the hits obtained (subsection 2.3), in order to recognise reliable structural and functional associations. Such an implementation of combination of profile-based techniques is not only capable of recognising reliable assignments but is also capable of enhancing residue coverage of the proteome in terms of recognisable structural and functional domains.

ii. Exploration of superfamily relationships: Some of the recognisable domains, albeit conserved across various species, lack clarity in their structural and/or functional aspects. We have made an attempt to address the structural and/or functional aspects of these uncharacterised protein families by means of their evolutionary relationship with wellcharacterised protein families. This exercise has also aided in recognising possible structure and/or function acquired by $M$. tuberculosis proteins comprising such uncharacterised domains.

iii. Assessment of reliability of the predictions: The criteria used to assess all the above relationships are detailed in this subsection.

iv. Exploring domain unassigned regions in proteins: A computationally intensive pipeline was used to recognise domains that may have been missed out in our primary steps.

v. Structure and function recognition for proteins with no recognisable domains: We have also made an attempt to investigate the proteins with no recognisable structural or functional domains using profile matching methods, for their possible evolutionary relationship with proteins of known structure and function. To achieve this, we have used fold recognition algorithm to determine probable fold acquired by the protein under question and a sensitive profile-based search tool for an iterative search against non-redundant sequence database to recognise homologues of known function.

Details on the techniques, in-house algorithms, protein family resources and derived databases used are elaborated further in this section. The protocol used is delineated in Figure-1. 


\subsection{Recognition of structural and functional domains}

\subsubsection{Datasets}

Multiple sequence alignments of sequence domain families were obtained from Pfam (v.26) [프] along with their corresponding HMM profiles. The multiple sequence alignments of various domain families were used as basis to generate a searchable database of multiple PSSM profiles, MulPSSM (http://mulpssm.mbu.iisc.ernet.in) [16, 17], for each domain family. These multiple PSSMs correspond to an alignment, where multiple sequences in a domain family are used as reference. Use of such multiple PSSMs has been shown to improve sensitivity of remote homologue detection [18]. In addition, benchmark studies [펴-36] have shown a reasonably good coverage of proteins for function annotation. The current version of MulPSSM database comprises of 385,258 profiles for 13,672 sequence domain families and 14,235 profiles corresponding to 3,856 structural families in PALI [15] database based on SCOP (version 1.75).

A database developed by one of our research groups known as TOCCATA (manuscript under preparation, http://www-cryst.bioc.cam.ac.uk/toccata), includes 8,151 statistical profiles generated using alignments of representative structures from SCOP (version 1.75A) families and CATH (version 3.5) [37] superfamilies which is searchable with a sensitive fold recognition program (referred as FUGUE) [12]. Complementing the work published by Mao et al. [్], a genome-scale structure prediction of gene products of $M$. tuberculosis with the help of a homology modelling pipeline involving use of profiles from TOCCATA, generated reliable structural models. A database dedicated for such models is CHOPIN (manuscript submitted, http://structure.bioc.cam.ac.uk/chopin) which was used to retrieve structural domain assignments for proteins in M. tuberculosis. Also, 15,438 profile HMMs were obtained for protein structural superfamilies from SUPERFAMILY. A database derived from HMM profile library of Pfam (v.26) was retrieved for an iterative sequence search using profile-profile alignment (HHblits; ftp://toolkit.genzentrum.lmu.de/pub/HH-suite/databases/hhsuite_dbs/) [24]].

\subsubsection{Techniques used to analyse gene products in M. tuberculosis}

The entire repertoire of proteins retrieved from TubercuList was first subjected to hmmscan, a program available through HMMER3.0 package [38], which scans each sequence against a collection of profile-HMMs. Profile-HMMs of domain families (13672) obtained from Pfam v.26 were used to assign domains. Structural domain assignments were derived from CHOPIN database. Additionally, a profile-based search exercise was accomplished for structural domain assignment, with use of profile-HMMs (15348) from SUPERFAMILY. RPS-BLAST [11], provided by NCBI 
BLAST package, was employed for the use of multiple PSSMs of various domain families to search against the protein sequences. HHblits, an iterative sequence search tool which makes use of HMMHMM alignment, is reportedly capable of generating accurate alignments with a sensitivity of 50$100 \%$ higher than the current profile-profile match approaches. HHblits was used for function recognition of proteins, with the help of a searchable HMM library of Pfam v.26.

\subsection{Exploration of superfamily relationships}

AlignHUSH (http://alignhush.mbu.iisc.ernet.in) [23], developed by one of our groups, is a highly sensitive HMM-HMM match algorithm capable of exploring relationships between sequencedomain families obtained from Pfam (v.26) and structural families obtained from SCOP (version 1.75). A database, thus generated, i.e. SUPFAM (http://supfam.mbu.iisc.ernet.in) [39] captures such relationships derived between sequence and structural families. In addition, an all-against-all profile-profile comparison amongst Pfam families of no known structural information has resulted in identification of potentially new superfamilies comprising of related Pfam families.

Furthermore, protein sequence-structure relationships derived from the CHOPIN database not only aided the inferences drawn on structural domain assignments, but also facilitated association of putative structural information to various uncharacterized protein families. In other words, the ability to model the structure of a protein housing an uncharacterized protein family could shed light on the probable structure acquired by the concerned protein family. Exploration of such relationships has also been of profound use for annotation of uncharacterized protein families identified in M. tuberculosis proteome.

\subsection{Assessment of reliability of the predictions}

Each of the associations made for a protein sequence was evaluated in terms of its statistical significance i.e. e-value. In the case of RPS-BLAST-based use of MulPSSM database, an e-value threshold of 0.01 was used to identify reliable domain assignments. A profile coverage cut-off of $70 \%$ was also used as an additional criterion to minimise the occurrence of false positives. For domain assignments using hmmscan against Pfam database, family-specific gathering thresholds (GA) obtained from Pfam were used to extract reliable domain assignments. Gathering thresholds are chosen in such a way to maximize the coverage of a family while excluding false positives [33]. These roughly correspond to an e-value cut-off of 0.01. Every domain family in Pfam is associated with two such thresholds i.e. sequence bit score and domain bit score. A protein sequence with a bit score more significant than that of the two GAs of a particular domain family is considered to be a reliable hit. For hits identified by HHblits, an e-value more significant than $1 \times 10^{-3}$ was used to 
associate domain families, based on benchmark studies on evaluation of true positives [24]. For structural domain associations derived from CHOPIN, Z-score values over 4.0 were considered significant and for structural domain associations identified using hmmscan against SUPERFAMILY database, an e-value cut-off of 0.0001 was used. Where all the above approaches identified similar domain family hits for the same region in a given protein sequence, the domains recognised by hmmscan were adopted. Domains obtained by the use of RPS-BLAST, HHblits and those derived from SUPERFAMILY and CHOPIN were taken into consideration for proteins when one of the approaches could identify a reliable domain family hit a) for a completely unassigned protein sequence, missed by hmmscan and b) for substantially long unassigned regions in proteins already associated with a domain family by hmmscan. The domain assignments for all the proteins in $M$. tuberculosis are thus manually curated to maximize residue and sequence coverage. During the course of analysis, the Pfam database in its recent release (v.27) was updated by about 1150 domain families. Attempts have been made to ensure that all the domain assignments concur with the current domain families in the recent release of Pfam.

For the assessment of relationships identified between sequence (Pfam v.26) and structural (SCOP v.1.75) families, a Z-score cut-off of 7.5 and a query coverage of greater than or equal to $60 \%$ was used [35]. Cases of a single Pfam family relating to more than one SCOP family were assessed for overlapping regions. Where no overlaps were observed, relationships between a Pfam family and more than one SCOP family were recognised. Overlaps were resolved using additional criteria: a) If the SCOP families relating to one Pfam family belong to the same superfamily, the family with the best Z-score is considered, b) If the SCOP families belong to the same fold, the superfamily with the best Z-score is considered. Stringent criteria were used to identify potentially new superfamilies which comprise of related Pfam families. A Z-score cut-off of 9.0 and a query coverage threshold of $80 \%$ were used. All the superfamily relationships were manually curated to ensure reliability of the associations made.

\subsection{Exploring domain unassigned regions in proteins}

If the methods mentioned above are not successful in recognising structural or functional domains, domain association was further explored using a computationally intensive pipeline, PURE (Prediction of Unassigned REgions) [40], developed earlier by one of our groups. Filters for the prediction of transmembrane regions and coiled-coils form the first step to ensure unassigned regions have a potential to be associated to pre-existing domain families. Subsequent filters ensure that the unassigned region under question has substantial predicted secondary structural content and evolutionary conservation. Starting from a set of homologous sequences of the query unassigned 
region and their domain architectures, it is usually possible to infer structure and function for the unassigned regions.

\subsection{Structure and function recognition for proteins with no recognizable domains}

Protein sequences for which the above methods were unsuccessful in annotation were subjected to fold recognition using PHYRE ${ }^{2}$ (http://www.sbg.bio.ic.ac.uk/phyre2/index.cgi). Proteins with newly identified domain assignments were also subjected to this approach. High confidence matches, i.e. $>90 \%$ were considered to ensure correctness of the recognised fold [41]. Also, proteins with no domain assignment were searched against non-redundant database from NCBI (ftp://ftp.ncbi.nih.gov/blast/db/) using HHblits with an e-value cut-off of $10^{-3}$ subjected to 8 rounds of iterations. Query coverage equal to or better than $60 \%$ was another criterion employed in order to eliminate short stretches of alignments. All the hits obtained were manually curated to ensure identification of reliable functional homologues.

\section{Results and Discussion}




\subsection{Overview on the analyses of proteins in M. tuberculosis}

A total of 3566 (89\%) out of 4018 gene products encoded in M. tuberculosis genome could be associated with at least one structural or functional domain assignment, of which 1891 (53\%) proteins have residue coverage greater than $80 \%$. A bar graph representation of the percentage residue coverage obtained for the proteins is illustrated in Figure-2. As depicted in the figure, residue coverage of $80-90 \%$ could be achieved for 533 proteins, while a residue coverage of $>90 \%$ could be achieved for 1358 proteins alone. The amino acid residue coverage achieved considering all the domain assignments is $64 \%$, which is higher (by 4\%) than the available proteome-wide domain annotation coverage in Pfam for $M$. tuberculosis. For the proteins with no domain assignment function recognition was aided with the help of HHblits, an iterative HMM-HMM search, against a non-redundant sequence database. Homologues of known function were thus identified for 205 gene products, of which functions for 98 gene products were determined based on our analysis and the rest (107) are consistent with predicted functions in TubercuList database. Also, measures were taken to assess the fold-ability of secondary structure elements for proteins with no domain assignments with the help of fold recognition algorithm PHYRE ${ }^{2}$. Folds could be recognised for 64 proteins by means of their association with a protein of known structure. This approach, however, implicates the predicted fold adopted by the protein and transfer of a function is often not the case. Of the remaining 183 (5\%) gene products with no identified structure/function, 165 are prevalent in mycobacteria and 18 gene products are identified as pathogen specific and some of these are predicted to be involved in virulence [4]. Figure-3 summarizes these particularisations for 4018 gene products.

Pfam, a collection of sequence domain families, is also comprised of DUFs or Domains of Unknown Function that are large sets of functionally uncharacterized protein domain families and in many cases structurally uncharacterized as well [42]. Gene products encoded in M. tuberculosis constitute 268 such DUFs/UPFs. Exploration of relationships between DUFs and well-characterized protein families can provide useful insights in recognition of probable structure and function for DUFs. With the help of SUPFAM database and the details on structural hits derived from CHOPIN, 2\% (Supplementary Table-1) of Pfam domain families could be associated with known structural families, thus enhancing the structural content of the proteome. Association of structures with functionally characterized protein families of no known structural information is also reported in our analysis.

\subsection{Analyses on domain families identified in M. tuberculosis}




\subsubsection{Abundant domain families in the pathogen}

Frequency of occurrence of various domain families in $M$. tuberculosis proteome was analysed. Supplementary Figure-1 shows a histogram representation of the observed frequencies of the top 15 most populated domain families in M. tuberculosis, along with the number of gene products in which these domain families occur. The high occurrence of domain families playing an important role in host-pathogen interactions (PE and PPE) [43-45]; multidrug resistance and pathogenicity (bacterial regulatory proteins tetR family and PIN) [46-48]; drug-efflux pumps (ABC and MFS transporters) [49-51]; lipid metabolism (Acyl-CoA dehydrogenases and PP-binding) and eliciting inappropriate immune response [드] (short-chain dehydrogenases, adh_short) have been well documented previously by one of our groups []ㅡ ] and are present in the proteome annotations in Pfam database as well.

3.2.2 Domain families specific in $M$. tuberculosis as compared to those in human proteome

The domain families and superfamilies in M. tuberculosis were compared with those observed in gene products encoded in the human genome, and domain families which are abundant and specific to the pathogen were assessed. The domain definitions of the proteins encoded in human genome were obtained from Pfam database. Table 1 lists the most frequently occurring domain families in M. tuberculosis that are absent in the human proteome. The entire list of domain families identified in M. tuberculosis, absent in human proteome, is provided in Supplementary Table-2.

Domain assignments for previously un-annotated and incompletely annotated gene products (45 gene products), whose domain families are specific to the pathogen are highlighted in Supplementary Table-3a. For instance, $R v 2798 c$, a conserved hypothetical protein of 108 amino acids, is predicted to comprise of a WXG100 domain of the ESAT-6-like family, at an e-value of $9 \times 10^{-16}$ in the region 2-79 of the protein. As depicted in Table 1, the WXG100 domain family, absent in the human proteome, represents a secretion system which is postulated to play a key role in virulence and protective immunity in M. tuberculosis [53]. The domain prediction also concurred with predictions derived using CHOPIN database wherein ESAT-6-like structural family could be associated with a Z-score of 4.38 in the region 8-82 of the hypothetical protein. A list of 3566 gene products with all the domain definitions is provided in Supplementary Table-3a.

564 domain families identified in 1093 gene products of the pathogen are absent in the human proteome (Supplementary Table-2). Some domains, such as those functioning as virulence factors 
involved in host-pathogen interaction such as immunodominance, aiding entry and survival in host macrophages, are highly represented. The absence of such domain families in the host proteome suggests that proteins harbouring these domains may be attractive drug targets.

\subsection{3 “New” domain assignments}

Domain assignments obtained for completely unassigned proteins and for domain unassigned regions present in annotated proteins are termed new domain assignments in our analysis. For 157 domain unassigned proteins and for 87 proteins with domain unassigned regions, 205 domain families could be assigned.

Consensus results obtained from multiple independent approaches indicated reliability of the predictions made. Structural information obtained for $244(157+87)$ gene products using PHYRE ${ }^{2}$ corroborate nearly all the remote homology relationships identified using profile-based approaches. Also, the protein functions predicted by the curators, for feasible cases in TubercuList database, were consistent with domain assignments made. Table 2 summarizes a few of the new domain assignments obtained. The potential biological roles corresponding to the domain assignments for two of the gene products are described further.

Rv3630, a protein of 431 amino acids, is identified to comprise of a multi-antimicrobial extrusion domain (MatE). Proteins harbouring this domain represent a family of transport proteins involved in mediating resistance to a wide range of cationic dyes, aminoglycosides and fluoroquinolones via proton motive force dependent efflux mechanisms [ [54]. These drug/sodium antiporters usually consist of 12 transmembrane regions, consistent with the 12-helix bundle fold predicted using PHYRE ${ }^{2}$ and shown in Figure-4(a). Initially predicted to be an integral membrane protein of unknown function [4], the probable function of Rv3630 is identified as a multidrug transport protein.

$R v 0268 c$, a conserved hypothetical protein of 169 amino acids, has been predicted to constitute a domain known to act as an antitoxin in type II toxin-antitoxin systems (PhdYeFM_antitox) which is predominant in bacteria. This domain family belongs to a class of plasmid encoded toxins that are primarily involved in plasmid maintenance in the host by means of post-segregational cell killing system where loss of the plasmid results in activation of toxins and inactivation of antitoxins [46]. Accumulation of toxins thereby, triggers the production of antitoxins, which when bound to their toxin partners act as regulatory switches to repress the expression of operons encoding toxin and antitoxin genes [55].The DNA-binding activity of Phd antitoxins has been well documented 
previously [ $[\underline{6}, \underline{57}]$ wherein the N-terminus of Phd antitoxin is essential for DNA-binding and Cterminus is essential for antitoxin activity. Furthermore, presence of antiparallel $\beta$-sheet in the $\mathrm{N}$ terminal region of Phd antitoxin is believed to facilitate specific contacts in the major groove of DNA [56]. Consistent with this observation the N-terminal region of the predicted antitoxin domain in $R v 0268 c$ comprises of an antiparallel $\beta$-sheet as depicted in Figure-4(b) which shows the predicted secondary structural elements. Fold prediction results for Rv0268c yielded a high confidence match (>90\%) with the antitoxin relF from $M$. tuberculosis. Figure-4(c) illustrates the crystal structure of toxin-antitoxin complex relBF [Miallau L., Chernishof I., Chiang J., Arbing M., Cascio D., and Eisenberg D.; to be published according to Protein Data Bank (PDB)] from M. tuberculosis, where the antitoxin component is shown in green. Formerly recognised as a conserved hypothetical protein, $R v 0268 \mathrm{c}$ could be a putative antitoxin.

A list of all the new domain assignments for 244 gene products in $M$. tuberculosis proteome is provided in Supplementary Table-3b.

\subsubsection{Characterizing the uncharacterized domain families}

A total of 1932 structural and functional domain families could be associated with $89 \%$ of $4018 \mathrm{M}$. tuberculosis proteins, which include 268 DUFs/UPFs. Pfam, a comprehensive collection of sequence domain families, includes DUFs that are large sets of functionally uncharacterized domain families. A substantial proportion of DUFs in the Pfam database are structurally uncharacterized as well. Numerous attempts have been made in the past to "de-DUF” a DUF, and have been successful in identifying functions for such families [58-62]. Employment of the highly sensitive profileprofile match approach, AlignHUSH, has facilitated exploration of structural and functional relationships between uncharacterized and well-characterized domain families. SUPFAM database comprehends such relationships derived using AlignHUSH. With the help of SUPFAM database, a total of 55 Pfam domain families in M. tuberculosis could be either associated with known structural families or could be related to at least one Pfam family from the Pfam database thus representing potentially new Pfam superfamilies. These Pfam superfamilies can be regarded as analogous to Pfam clans which are groups of related Pfam domain families based on similarity of sequence, structure or profile-HMM [63]. Figure-5 shows a pie representation of the structural content pertaining to Pfam domain families in M. tuberculosis proteome. An increase of 2\% (34 domain families) in the structural content of the proteome is achieved with the help of superfamily relationships as shown in the figure. Exploring such domain family relationships also aids in recognition of probable structures and/or functions for the protein housing the domain family concerned; these may have been missed by conventional sequence-based search algorithms. 
A total of 34 Pfam domain families (including 15 DUFs/UPFs) could be associated with known structural families and 21 Pfam domain families (including 11 DUFs/UPFs) could be associated with potentially new Pfam superfamilies. These associations are summarized for a few cases in Table 3 and the entire list is included in supplementary information (Supplementary Table 1 and 4). The analysis was initially done with an earlier release of Pfam (Pfam v.26); where close to 4\% of the domain families in $M$. tuberculosis with no structural information were associated to known structural families. Several predicted associations successfully coincided with the updated information on domain families in the recent release of Pfam (v.27), highlighted in italics in Table 3. Structural and functional inferences drawn for few cases are discussed further in depth.

A set of two conserved hypothetical proteins $R v 1754 c$ and $R v 3707 c$, members of DUF4185 family, could be associated with structural superfamily of sialidases. Sialidases belong to a family of glycoside hydrolases, and are involved in hydrolysis of glycosidic linkages of terminal sialic residues in carbohydrate moieties such as glycolipids. Sialidases have also been implicated in pathogenesis in several Gram positive bacteria [64]. Since there is no apparent sialidase or neuraminidase activity observed in mycobacteria [ㄷ] DUF4185 members $R v 1754 c$ and $R v 3707 c$ in M. tuberculosis may not function as sialidases. A family of 408 members, DUF4185 predominantly comprises of bacterial proteins. Fold-prediction demonstrated that more than $90 \%$ of the members of DUF4185 family show high confidence matches (95-100\%) with glycoside hydrolases. Glycoside hydrolases are a widespread group of enzymes, classified into more than 100 families based on their sequence similarities [66-68], where the varied substrate specificities and the mode of action of enzymes in these different families can be attributed to differences in the details of their structures rather than by their overall fold [67]. Illustrated in Figure-6 is the 6-bladed beta-propeller fold, similar to sialidases, adopted by members of DUF4185 family. As discussed previously, due to the absence of sialidases in mycobacteria, $R v 1754 c$ and $R v 3707 c$ constituting DUF4185 family could only be structurally associated to sialidases, whilst other non-mycobacterial members in DUF4185 family could probably function as sialidases based on the conservation of residues essential for sialidase activity. Further experimental investigations on $R v 1754 \mathrm{C}$ and $R v 3707 \mathrm{C}$ could provide elucidations on the nature of their potential glycoside hydrolase activity.

$R v 3605 c$, a probable conserved secreted or membrane protein [4] consisting of a domain of unknown function, DUF3180, could be related to the tripartite tricarboxylate transporter TctB family. This case highlights the association of a DUF family with a family of known function but unknown structure. Formerly a domain of unknown function, TctB transporter family comprises of members, some of which are annotated as TctB subunit of tripartite tricarboxylate transporter family 
having 4 transmembrane regions, as reported in Pfam database. M. tuberculosis is not known to code for a protein functioning as tripartite tricarboxylate transporter. However, the presence of membrane spanning regions identified in members of DUF3180 family with use of fold-recognition approach corroborates our predicted association of this family with a transporter.

Rv2395, a conserved integral membrane protein predicted to be involved in transport [4], contains an oligopeptide transporter domain (OPT). These transporters are thought to comprise of 12-14 transmembrane regions distinct from ATP-binding cassette (ABC) transporter and protondependent oligopeptide transporter (PTR) families [69]. Based on our analysis, the OPT domain family of unknown structure could be associated with a structural superfamily, MFS general substrate transporter which comprises of 12 transmembrane regions with structurally similar $\mathrm{N}$ - and C-terminal halves. The structural and functional inferences thus drawn for domains of unknown structure/function, based on exploration of superfamily relationships using SUPFAM database, can provide useful insights into the structure and the functions of the proteins containing the domain family.

\subsection{Structure recognition for proteins with no recognisable domains}

Fold-recognition using PHYRE ${ }^{2}$ was also employed on the set of gene products in M. tuberculosis for which no domains could be assigned (452 gene products). Such an exercise aided in assessment of foldability of the secondary structure elements in such proteins, primarily based on the observation that the number of folds in nature is limited and many remotely homologous protein sequences, although functionally disparate, can preferably adopt similar structures. Thus, transfer of a function based on the fold prediction alone is not always the case. 64 of 452 gene products with no known domain assignment were predicted to adopt a structure, which constitutes $2 \%$ of the total proteome as depicted in Figure-3. To ensure correctness of the model, only high confidence matches (>90\%) were considered for predicting fold of a protein based on benchmarked accuracy criteria in

PHYRE $^{2}$. Given a high confidence match, the overall fold is likely to be correct and the central core of the model tends to be accurate even at low sequence identities $(<20 \%)[\underline{41}, \underline{70}]$. Table 4 lists a few of the proteins predicted to assume a known fold. Supplementary Table- 5 provides the entire list of 64 gene products and information on their predicted folds.

\subsection{Function recognition for proteins with no recognisable domains}

Function recognition for the remaining 388 proteins was aided with the help of an iterative sequence search against non-redundant sequence database. A total of 205 proteins were identified 
where the functions could be inferred from the functions of their homologues. Many of our predicted functional associations (107 gene products) concurred with the predicted functions reported in TubercuList database. For 98 proteins, we could infer putative functions based on homology. This list of 98 proteins and description of their homologues is provided in Supplementary Table-6.

\subsection{Analyses on hypothetical proteins}

M. tuberculosis proteome comprises of 1146 conserved hypothetical proteins constituting a significant $29 \%$ of the proteome [4]. A considerable number (151) of these experimentally uncharacterized gene products have been identified to be essential for either in vitro or in vivo growth of the pathogen [ $\underline{71-73]}$, thus gaining importance in structure and function prediction efforts. Attempts have been made to annotate such hypothetical proteins in M. tuberculosis using combination of bioinformatics predictions and experimentally determined properties. In the process, function prediction for a reasonable number of conserved hypotheticals has been already achieved [4]. Based on our analyses, domain annotations could be determined for 826 of 1146 hypothetical proteins encompassing 127 hypotheticals known to be essential for the pathogen. Our predictions concur with the partial information on predicted functions available in TubercuList database for feasible cases. Table 5 summarizes the domain assignments for hypothetical proteins known to be essential.

Furthermore, of the remaining 320, function/structure recognition for 125 hypothetical proteins could be achieved as depicted in Figure-7. The number of hypothetical proteins known to be essential for the pathogen is enlisted in polygons for each category in the figure. Identification of hypothetical proteins localised to membrane. Detailed predictions for each of the hypothetical protein are provided in three subsections in Supplementary Table-7. The next subsection highlights the feasible inferences on those gene products for which no reliable predictions could be achieved.

\subsection{The orphans: pathogen specific proteins}

For a total of 183 gene products, sensitive profile-based approaches were unsuccessful in identifying homologues. More than $90 \%$ of these were recognised as hypothetical proteins of unknown functions. However, putative functional information such as signalling peptides or secreted antigens, could be ascribed to 15 of these gene products, based on proteomic analysis [ $\underline{74}$ ] and presence of specific genomic islands [75]. 18 of 183 gene products could be identified as strain specific, which are enlisted in Table 6. Such pathogen-specific proteins could be further probed with the help of experimental efforts for their participation in virulence and pathogenesis. 
Ramakrishnan et.al 


\section{Conclusions}

An enriched structural and functional characterization of $M$. tuberculosis proteome is presented corresponding to $89 \%$ of 4018 gene products with 1932 domain assignments. Use of sensitive approaches have also aided in putative structure/function recognition for an additional 6\% (269 gene products) of the proteome.

Domain definitions identified for 244 proteins, which include previously domain unassigned proteins and annotated proteins with domain unassigned regions, provide a basis to re-explore their participation in various pathways involved in host-pathogen interactions. Profile-based methods have tremendous advantages owing to their high efficiency. Where direct functional inference was not possible, structure and/or function could be inferred by taking advantage of indirect methods and sensitive remote homology detection techniques, thus associating structural and functional information for 55 domain families of currently unknown structure or function. Such an exercise can deliver useful insights in understanding the probable structure or function acquired by the protein housing the domain family concerned. Improving sensitivity in detection of relationships through remote-homology based approaches as demonstrated enhances the usefulness significantly and provides functional inferences without the need for explicitly obtaining structural models. Attributing putative functions to 955 of 1146 hypothetical proteins can not only enhance the functional repertoire of the pathogen, but can also provide a suitable ground to explore their participation in pathogenesis. Pathogen-specific proteins (18), which might have potential implications in virulence, can very well serve as promising drug targets. The functional inferences drawn based on our predictions can thus provide valuable basis for experimental endeavours in understanding the intricate mechanisms in pathogenesis and underpinning the process of drug target discovery.

\section{Acknowledgements}

We thank Prof. Samir Brahmachari and Dr. Anshu Bharadwaj for their comments and encouragement.

\section{Funding}

This research was supported primarily by OSDD programme of CSIR and also by Mathematical Biology programme, Department of Science and Technology, Department of Biotechnology, India, and by the Bill and Melinda Gates Foundation. 


\section{Competing interests}

None declared 


\section{References}

1. Cole, S.T., R. Brosch, J. Parkhill, T. Garnier, C. Churcher, D. Harris, et al. Deciphering the biology of Mycobacterium tuberculosis from the complete genome sequence. Nature, 1998. 393(6685): p. 537-44.

2. Camus, J.C., M.J. Pryor, C. Medigue, and S.T. Cole. Re-annotation of the genome sequence of Mycobacterium tuberculosis H37Rv. Microbiology, 2002. 148(Pt 10): p. 2967-73.

3. Namboori, S., N. Mhatre, S. Sujatha, N. Srinivasan, and S.B. Pandit. Enhanced functional and structural domain assignments using remote similarity detection procedures for proteins encoded in the genome of Mycobacterium tuberculosis H37Rv. J Biosci, 2004. 29(3): p. 245-59.

4. Lew, J.M., A. Kapopoulou, L.M. Jones, and S.T. Cole. TubercuList--10 years after. Tuberculosis (Edinb), 2011. 91(1): p. 1-7.

5. Anand, P., S. Sankaran, S. Mukherjee, K. Yeturu, R. Laskowski, A. Bhardwaj, et al. Structural annotation of Mycobacterium tuberculosis proteome. PLoS One, 2011. 6(10): p. e27044.

6. Mao, C., M. Shukla, G. Larrouy-Maumus, F.L. Dix, L.A. Kelley, M.J. Sternberg, et al. Functional assignment of Mycobacterium tuberculosis proteome revealed by genome-scale fold-recognition. Tuberculosis (Edinb), 2013. 93(1): p. 40-6.

7. Vashisht, R., A.K. Mondal, A. Jain, A. Shah, P. Vishnoi, P. Priyadarshini, et al. Crowd sourcing a new paradigm for interactome driven drug target identification in Mycobacterium tuberculosis. PLoS One, 2012. 7(7): p. e39808.

8. Altschul, S.F., W. Gish, W. Miller, E.W. Myers, and D.J. Lipman. Basic local alignment search tool. J Mol Biol, 1990. 215(3): p. 403-10.

9. Park, J., K. Karplus, C. Barrett, R. Hughey, D. Haussler, T. Hubbard, et al. Sequence comparisons using multiple sequences detect three times as many remote homologues as pairwise methods. J Mol Biol, 1998. 284(4): p. 1201-10.

10. Altschul, S.F., T.L. Madden, A.A. Schaffer, J. Zhang, Z. Zhang, W. Miller, et al. Gapped BLAST and PSI-BLAST: a new generation of protein database search programs. Nucleic Acids Res, 1997. 25(17): p. 3389-402.

11. Marchler-Bauer, A., A.R. Panchenko, B.A. Shoemaker, P.A. Thiessen, L.Y. Geer, and S.H. Bryant. CDD: a database of conserved domain alignments with links to domain three-dimensional structure. Nucleic Acids Res, 2002. 30(1): p. 281-3.

12. Shi, J., T.L. Blundell, and K. Mizuguchi. FUGUE: sequence-structure homology recognition using environment-specific substitution tables and structuredependent gap penalties. J Mol Biol, 2001. 310(1): p. 243-57.

13. Eddy, S.R. Profile hidden Markov models. Bioinformatics, 1998. 14(9): p. 755-63.

14. Ye, J., S. McGinnis, and T.L. Madden. BLAST: improvements for better sequence analysis. Nucleic Acids Res, 2006. 34(Web Server issue): p. W6-9.

15. Balaji, S., S. Sujatha, S.S. Kumar, and N. Srinivasan. PALI-a database of Phylogeny and ALlgnment of homologous protein structures. Nucleic Acids Res, 2001. 29(1): p. 61-5.

16. Anand, B., V.S. Gowri, and N. Srinivasan. Use of multiple profiles corresponding to a sequence alignment enables effective detection of remote homologues. Bioinformatics, 2005. 21(12): p. 2821-6.

17. Gowri, V.S., O. Krishnadev, C.S. Swamy, and N. Srinivasan. MulPSSM: a database of multiple position-specific scoring matrices of protein domain families. Nucleic Acids Res, 2006. 34(Database issue): p. D243-6.

18. Gowri, V.S., K.G. Tina, O. Krishnadev, and N. Srinivasan. Strategies for the effective identification of remotely related sequences in multiple PSSM search approach. Proteins, 2007. 67(4): p. 789-94. 
19. Sandhya, S., S. Chakrabarti, K.R. Abhinandan, R. Sowdhamini, and N. Srinivasan. Assessment of a rigorous transitive profile based search method to detect remotely similar proteins. J Biomol Struct Dyn, 2005. 23(3): p. 283-98.

20. Sandhya, S., R. Mudgal, C. Jayadev, K.R. Abhinandan, R. Sowdhamini, and N. Srinivasan. Cascaded walks in protein sequence space: use of artificial sequences in remote homology detection between natural proteins. Mol Biosyst, 2012. 8(8): p. 2076-84.

21. Biegert, A. and J. Soding. Sequence context-specific profiles for homology searching. Proc Natl Acad Sci U S A, 2009. 106(10): p. 3770-5.

22. Soding, J. Protein homology detection by HMM-HMM comparison. Bioinformatics, 2005. 21(7): p. 951-60.

23. Krishnadev, O. and N. Srinivasan. AlignHUSH: alignment of HMMs using structure and hydrophobicity information. BMC Bioinformatics, 2011. 12: p. 275.

24. Remmert, M., A. Biegert, A. Hauser, and J. Soding. HHblits: lightning-fast iterative protein sequence searching by HMM-HMM alignment. Nat Methods, 2012. 9(2): p. 173-5.

25. Chothia, C. and A.M. Lesk. The relation between the divergence of sequence and structure in proteins. EMBO J, 1986. 5(4): p. 823-6.

26. Chothia, C. and M. Gerstein. Protein evolution. How far can sequences diverge? Nature, 1997. 385(6617): p. 579, 81.

27. Reid, A.J., C. Yeats, and C.A. Orengo. Methods of remote homology detection can be combined to increase coverage by $10 \%$ in the midnight zone. Bioinformatics, 2007. 23(18): p. 2353-60.

28. Sonnhammer, E.L., S.R. Eddy, and R. Durbin. Pfam: a comprehensive database of protein domain families based on seed alignments. Proteins, 1997. 28(3): p. 405-20.

29. Gough, J., K. Karplus, R. Hughey, and C. Chothia. Assignment of homology to genome sequences using a library of hidden Markov models that represent all proteins of known structure. J Mol Biol, 2001. 313(4): p. 903-19.

30. Murzin, A.G., S.E. Brenner, T. Hubbard, and C. Chothia. SCOP: a structural classification of proteins database for the investigation of sequences and structures. J Mol Biol, 1995. 247(4): p. 536-40.

31. Lewis, S., M. Ashburner, and M.G. Reese. Annotating eukaryote genomes. Curr Opin Struct Biol, 2000. 10(3): p. 349-54.

32. Li, W.W., G.B. Quinn, N.N. Alexandrov, P.E. Bourne, and I.N. Shindyalov. A comparative proteomics resource: proteins of Arabidopsis thaliana. Genome Biol, 2003. 4(8): p. R51.

33. Punta, M., P.C. Coggill, R.Y. Eberhardt, J. Mistry, J. Tate, C. Boursnell, et al. The Pfam protein families database. Nucleic Acids Res, 2012. 40(Database issue): p. D290-301.

34. Gowri, V.S. Analysis of protein evolution and its implications in remote homology detection and function recognition., in Molecular Biophysics Unit2007, Indian Institute of Science: Bangalore.

35. Krishnadev, O. Development and applications of computational methods to aid recognition of protein functions and interactions., in Molecular Biophysics Unit2010, Indian Institute of Science: Bangalore.

36. Mohanty, S. Functionally interacting proteins: analyses and predictions, in Molecular Biophysics Unit2012, Indian Institute of Science: Bangalore.

37. Orengo, C.A., A.D. Michie, S. Jones, D.T. Jones, M.B. Swindells, and J.M. Thornton. CATH--a hierarchic classification of protein domain structures. Structure, 1997. 5(8): p. 1093-108.

38. Eddy, S.R. Accelerated Profile HMM Searches. PLoS Comput Biol, 2011. 7(10): p. e1002195.

39. Pandit, S.B., D. Gosar, S. Abhiman, S. Sujatha, S.S. Dixit, N.S. Mhatre, et al. SUPFAM--a database of potential protein superfamily relationships derived by comparing sequence-based and structure-based families: implications for 
structural genomics and function annotation in genomes. Nucleic Acids Res, 2002. 30(1): p. 289-93.

40. Reddy, C.C., K. Shameer, B.O. Offmann, and R. Sowdhamini. PURE: a webserver for the prediction of domains in unassigned regions in proteins. BMC Bioinformatics, 2008. 9: p. 281.

41. Bennett-Lovsey, R.M., A.D. Herbert, M.J. Sternberg, and L.A. Kelley. Exploring the extremes of sequence/structure space with ensemble fold recognition in the program Phyre. Proteins, 2008. 70(3): p. 611-25.

42. Bateman, A., P. Coggill, and R.D. Finn. DUFs: families in search of function. Acta Crystallogr Sect F Struct Biol Cryst Commun, 2010. 66(Pt 10): p. 1148-52.

43. Strong, M., M.R. Sawaya, S. Wang, M. Phillips, D. Cascio, and D. Eisenberg. Toward the structural genomics of complexes: crystal structure of a PE/PPE protein complex from Mycobacterium tuberculosis. Proc Natl Acad Sci U S A, 2006. 103(21): p. 8060-5.

44. Bachhawat, N. and B. Singh. Mycobacterial PE_PGRS proteins contain calciumbinding motifs with parallel beta-roll folds. Genomics Proteomics Bioinformatics, 2007. 5(3-4): p. 236-41.

45. Abdallah, A.M., N.D. Savage, M. van Zon, L. Wilson, C.M. Vandenbroucke-Grauls, N.N. van der Wel, et al. The ESX-5 secretion system of Mycobacterium marinum modulates the macrophage response. J Immunol, 2008. 181(10): p. 7166-75.

46. Anantharaman, V. and L. Aravind. New connections in the prokaryotic toxinantitoxin network: relationship with the eukaryotic nonsense-mediated RNA decay system. Genome Biol, 2003. 4(12): p. R81.

47. Dover, L.G., P.E. Corsino, I.R. Daniels, S.L. Cocklin, V. Tatituri, G.S. Besra, et al. Crystal structure of the TetR/CamR family repressor Mycobacterium tuberculosis EthR implicated in ethionamide resistance. J Mol Biol, 2004. 340(5): p. 1095105.

48. Arcus, V.L., P.B. Rainey, and S.J. Turner. The PIN-domain toxin-antitoxin array in mycobacteria. Trends Microbiol, 2005. 13(8): p. 360-5.

49. Pao, S.S., I.T. Paulsen, and M.H. Saier, Jr. Major facilitator superfamily. Microbiol Mol Biol Rev, 1998. 62(1): p. 1-34.

50. Braibant, M., P. Gilot, and J. Content. The ATP binding cassette (ABC) transport systems of Mycobacterium tuberculosis. FEMS Microbiol Rev, 2000. 24(4): p. 449-67.

51. De Rossi, E., P. Arrigo, M. Bellinzoni, P.A. Silva, C. Martin, J.A. Ainsa, et al. The multidrug transporters belonging to major facilitator superfamily in Mycobacterium tuberculosis. Mol Med, 2002. 8(11): p. 714-24.

52. Gamieldien, J., A. Ptitsyn, and W. Hide. Eukaryotic genes in Mycobacterium tuberculosis could have a role in pathogenesis and immunomodulation. Trends Genet, 2002. 18(1): p. 5-8.

53. Pallen, M.J. The ESAT-6/WXG100 superfamily -- and a new Gram-positive secretion system? Trends Microbiol, 2002. 10(5): p. 209-12.

54. Brown, M.H., I.T. Paulsen, and R.A. Skurray. The multidrug efflux protein NorM is a prototype of a new family of transporters., 1999, Blackwell Science Ltd, Molecular Microbiology: New South Wales, Australia. p. 393-5.

55. Garcia-Pino, A., S. Balasubramanian, L. Wyns, E. Gazit, H. De Greve, R.D. Magnuson, et al. Allostery and intrinsic disorder mediate transcription regulation by conditional cooperativity. Cell, 2010. 142(1): p. 101-11.

56. Smith, J.A. and R.D. Magnuson. Modular organization of the Phd repressor/antitoxin protein. J Bacteriol, 2004. 186(9): p. 2692-8.

57. Gerdes, K., S.K. Christensen, and A. Lobner-Olesen. Prokaryotic toxin-antitoxin stress response loci. Nat Rev Microbiol, 2005. 3(5): p. 371-82.

58. Martzen, M.R., S.M. McCraith, S.L. Spinelli, F.M. Torres, S. Fields, E.J. Grayhack, et al. A biochemical genomics approach for identifying genes by the activity of their products. Science, 1999. 286(5442): p. 1153-5. 
59. Schulze-Gahmen, U., J. Pelaschier, H. Yokota, R. Kim, and S.H. Kim. Crystal structure of a hypothetical protein, TM841 of Thermotoga maritima, reveals its function as a fatty acid-binding protein. Proteins, 2003. 50(4): p. 526-30.

60. Karras, G.I., G. Kustatscher, H.R. Buhecha, M.D. Allen, C. Pugieux, F. Sait, et al. The macro domain is an ADP-ribose binding module. EMBO J, 2005. 24(11): p. 1911-20.

61. Dlakic, M. DUF283 domain of Dicer proteins has a double-stranded RNA-binding fold. Bioinformatics, 2006. 22(22): p. 2711-4.

62. Krishna, S.S., L. Tautz, Q. Xu, D. McMullan, M.D. Miller, P. Abdubek, et al. Crystal structure of NMA1982 from Neisseria meningitidis at 1.5 angstroms resolution provides a structural scaffold for nonclassical, eukaryotic-like phosphatases. Proteins, 2007. 69(2): p. 415-21.

63. Finn, R.D., J. Mistry, B. Schuster-Bockler, S. Griffiths-Jones, V. Hollich, T. Lassmann, et al. Pfam: clans, web tools and services. Nucleic Acids Res, 2006. 34(Database issue): p. D247-51.

64. Rothe, B., B. Rothe, P. Roggentin, and R. Schauer. The sialidase gene from Clostridium septicum: cloning, sequencing, expression in Escherichia coli and identification of conserved sequences in sialidases and other proteins. Mol Gen Genet, 1991. 226(1-2): p. 190-7.

65. Arden, S.B., W.H. Chang, and L. Barksdale. Distribution of neuraminidase and nacetylneuraminate lyase activities among corynebacteria, mycobacteria, and nocardias. J Bacteriol, 1972. 112(3): p. 1206-12.

66. Henrissat, B. A classification of glycosyl hydrolases based on amino acid sequence similarities. Biochem J, 1991. 280 ( Pt 2): p. 309-16.

67. Davies, G. and B. Henrissat. Structures and mechanisms of glycosyl hydrolases. Structure, 1995. 3(9): p. 853-9.

68. Henrissat, B. and A. Bairoch. Updating the sequence-based classification of glycosyl hydrolases. Biochem J, 1996. 316 ( Pt 2): p. 695-6.

69. Lubkowitz, M.A., L. Hauser, M. Breslav, F. Naider, and J.M. Becker. An oligopeptide transport gene from Candida albicans. Microbiology, 1997. 143 ( Pt 2): p. 387-96.

70. Kelley, L.A. and M.J. Sternberg. Protein structure prediction on the Web: a case study using the Phyre server. Nat Protoc, 2009. 4(3): p. 363-71.

71. Sassetti, C.M., D.H. Boyd, and E.J. Rubin. Genes required for mycobacterial growth defined by high density mutagenesis. Mol Microbiol, 2003. 48(1): p. 7784.

72. Sassetti, C.M. and E.J. Rubin. Genetic requirements for mycobacterial survival during infection. Proc Natl Acad Sci U S A, 2003. 100(22): p. 12989-94.

73. Griffin, J.E., J.D. Gawronski, M.A. Dejesus, T.R. loerger, B.J. Akerley, and C.M. Sassetti. High-resolution phenotypic profiling defines genes essential for mycobacterial growth and cholesterol catabolism. PLoS Pathog, 2011. 7(9): p. e1002251.

74. de Souza, G.A., N.A. Leversen, H. Malen, and H.G. Wiker. Bacterial proteins with cleaved or uncleaved signal peptides of the general secretory pathway. J Proteomics, 2011. 75(2): p. 502-10.

75. Becq, J., M.C. Gutierrez, V. Rosas-Magallanes, J. Rauzier, B. Gicquel, O. Neyrolles, et al. Contribution of horizontally acquired genomic islands to the evolution of the tubercle bacilli. Mol Biol Evol, 2007. 24(8): p. 1861-71.

76. The PyMOL Molecular Graphics System, version 1.4.1 Schrodinger, L.L.C. 
Tables:

Table 1: List of some of the populated domain families in $M$. tuberculosis, absent in human proteome

\begin{tabular}{|c|c|c|c|}
\hline Pfam Domain Family & $\begin{array}{l}\text { Profile } \\
\text { Length }\end{array}$ & $\begin{array}{l}\text { No. of } \\
\text { occurrences }\end{array}$ & Description \\
\hline Pentapeptide repeats & 40 & 292 & Pentapeptide repeats. Function is unclear. \\
\hline $\mathrm{PE}$ & 94 & 89 & $\begin{array}{l}\text { Characteristic Pro-Glu (PE) motif with variable C- } \\
\text { terminus. Related to antigenic variation in } \\
\text { pathogen. }\end{array}$ \\
\hline PPE & 159 & 64 & $\begin{array}{l}\text { Characteristic Pro-Pro-Glu (PPE) motif with } \\
\text { variable C-terminus. Related to antigenic variation } \\
\text { in pathogen }\end{array}$ \\
\hline PE_PPE_C & 83 & 27 & $\begin{array}{l}\text { Conserved SVP motif. Implicated in } \\
\text { immunostimulation and virulence }\end{array}$ \\
\hline $\begin{array}{l}\text { Mammalian cell entry (mce) } \\
\text { proteins }\end{array}$ & 81 & 24 & Colonization and survival within macrophage \\
\hline $\begin{array}{l}\text { Protein of } 100 \text { residues with } \\
\text { WXG (WXG100) }\end{array}$ & 86 & 23 & $\begin{array}{l}\text { Important in virulence and protective immunity in } \\
\text { the pathogen }\end{array}$ \\
\hline
\end{tabular}


Table 2: Some of the new domain assignments in gene products of $M$. tuberculosis

\begin{tabular}{|c|c|c|c|c|}
\hline Gene ID & $\begin{array}{l}\text { Protein } \\
\text { length }\end{array}$ & Domain Family & $\begin{array}{l}\text { Domain } \\
\text { Region }\end{array}$ & E-value \\
\hline Rv0268c & 169 & $\begin{array}{l}\text { Antitoxin, type II toxin-antitoxin } \\
\text { system (PhDYeFM_antitox) }\end{array}$ & 88-152 & $3.5 \times 10^{-15}$ \\
\hline Rv0724A & 111 & $\begin{array}{l}\text { S-adenosyl-L-methionine-dependent } \\
\text { methyltransferases }\end{array}$ & $2-104$ & $7.06 \times 10^{-10}$ \\
\hline Rv2006 & 1327 & Hydrolase & $38-220$ & $3 \times 10^{-11}$ \\
\hline Rv2156c & 359 & $\begin{array}{l}\text { Phospho-N-acetylmuramoyl- } \\
\text { pentapeptide-transferase signature } 1 \\
\text { (MraY_sig1) }\end{array}$ & $46-58$ & $3.5 \times 10^{-8}$ \\
\hline Rv3630 & 431 & $\begin{array}{l}\text { Multi-antimicrobial extrusion protein } \\
\text { (MatE) }\end{array}$ & $42-184$ & $6.4 \times 10^{-8}$ \\
\hline
\end{tabular}


Table 3: Structural/functional associations obtained for Pfam domain families in $M$. tuberculosis using superfamily relationships.

\begin{tabular}{|c|c|c|c|c|c|c|}
\hline $\begin{array}{l}\text { Sr. } \\
\text { No. }\end{array}$ & Gene ID & $\begin{array}{l}\text { Pfam Domain } \\
\text { Family }\end{array}$ & $\begin{array}{l}\text { Domain } \\
\text { Region }\end{array}$ & $\begin{array}{l}\text { Structural/functional } \\
\text { association }\end{array}$ & Description & $\begin{array}{l}\text { Z-score based } \\
\text { on HMM- } \\
\text { HMM } \\
\text { matches using } \\
\text { AlignHUSH }\end{array}$ \\
\hline \multirow[t]{2}{*}{1.} & Rv2561 & \multirow[t]{2}{*}{ DUF2652 } & $34-74$ & \multirow{2}{*}{$\begin{array}{l}\text { SCOP superfamily, } \\
\text { d.58.29 }\end{array}$} & \multirow[t]{2}{*}{ Nucleotide cyclase } & \multirow[t]{2}{*}{7.6} \\
\hline & Rv2562 & & $1-33$ & & & \\
\hline \multirow[t]{2}{*}{2.} & Rv1754c & \multirow[t]{2}{*}{ DUF4185 } & $230-560$ & \multirow{2}{*}{$\begin{array}{l}\text { SCOP superfamily, } \\
\text { b.68.1 }\end{array}$} & \multirow[t]{2}{*}{ Sialidases } & \multirow[t]{2}{*}{10.9} \\
\hline & Rv3707c & & $5-327$ & & & \\
\hline 3. & Rv3605c & DUF3180 & $9-147$ & Pfam family, TctB & $\begin{array}{l}\text { Tripartite } \\
\text { tricarboxylate } \\
\text { transporter }\end{array}$ & 41.1 \\
\hline 4. & Rv2395 & $\begin{array}{l}\text { Oligopeptide } \\
\text { transporter } \\
\text { (OPT) }\end{array}$ & $10-605$ & $\begin{array}{l}\text { SCOP superfamily, } \\
\text { f.38.1 }\end{array}$ & $\begin{array}{l}\text { MFS general } \\
\text { substrate } \\
\text { transporter }\end{array}$ & 21.5 \\
\hline \multirow[t]{2}{*}{5.} & $R v 3259$ & \multirow[t]{2}{*}{ DUF1025 } & $76-138$ & SCOP superfamily, & \multirow{2}{*}{$\begin{array}{l}\text { Metalloproteases } \\
\text { (“zincins”), } \\
\text { catalytic domain }\end{array}$} & \multirow[t]{2}{*}{7.6} \\
\hline & $R v 3836$ & & $29-115$ & d.92.1 & & \\
\hline \multirow[t]{2}{*}{6.} & $R v 2018$ & \multirow[t]{2}{*}{ DUF433 } & $\begin{array}{l}183- \\
236\end{array}$ & \multirow{2}{*}{$\begin{array}{l}\text { SCOP superfamily, } \\
\text { a.4.1 }\end{array}$} & \multirow[t]{2}{*}{ Homeodomain-like } & \multirow[t]{2}{*}{28.8} \\
\hline & Rv2308 & & $\begin{array}{l}173- \\
227\end{array}$ & & & \\
\hline
\end{tabular}


Table 4: Folds predicted for the gene products with no domain assignment.

\begin{tabular}{|c|c|c|c|c|}
\hline Gene ID & Template description & $\begin{array}{l}\text { Model } \\
\text { confidence } \\
(\%)\end{array}$ & $\begin{array}{l}\text { Sequence } \\
\text { Identity (\%) }\end{array}$ & $\begin{array}{l}\text { Query } \\
\text { coverage }\end{array}$ \\
\hline Rv0965c & ESAT-6 like protein & 95.8 & 18 & $89 / 139$ \\
\hline Rv1950c & Virulence factor esxA & 91 & 15 & $46 / 63$ \\
\hline Rv3635 & $\begin{array}{l}\text { Oligosaccharide transferase } \\
\text { to N-glycosylate proteins }\end{array}$ & 99.1 & 10 & $405 / 591$ \\
\hline Rv3662c & $\begin{array}{l}\text { Fic domain containing } \\
\text { transcriptional regulator }\end{array}$ & 100 & 16 & $249 / 256$ \\
\hline
\end{tabular}


Table 5: List of domain annotations for hypothetical proteins identified to be essential in $M$. tuberculosis

\begin{tabular}{|c|c|c|c|c|c|}
\hline Gene ID & Gene essentiality & $\begin{array}{l}\text { Pfam } \\
\text { domain } \\
\text { family }\end{array}$ & $\begin{array}{l}\text { Domain } \\
\text { region }\end{array}$ & E-value & Description \\
\hline Rv0123 & $\begin{array}{l}\text { Essential for in } \\
\text { vitro growth }\end{array}$ & RHH_1 & $75-113$ & $3.4 \times 10^{-10}$ & $\begin{array}{l}\text { Ribbon-helix-helix, copG family, } \\
\text { protein repressor }\end{array}$ \\
\hline Rv0207c & $\begin{array}{l}\text { Essential for } \\
\text { survival in } \\
\text { primary murine } \\
\text { macrophages } \\
\text { (TraSH) }\end{array}$ & NYN & $39-203$ & $1 \times 10^{-6}$ & $\begin{array}{l}\text { Nedd4-binding protein and } \\
\text { bacterial YacP-like protein (NYN) } \\
\text { family comprise RNAses with a } \\
\text { PIN domain-like fold }\end{array}$ \\
\hline Rv1502 & $\begin{array}{l}\text { Essential for in } \\
\text { vitro growth }\end{array}$ & DUF377 & $70-298$ & $4 \times 10^{-6}$ & $\begin{array}{ll}\text { Predicted glycosyl hydrolases } \\
\text { belonging to beta-fructosidase } \\
\text { superfamily }\end{array}$ \\
\hline Rv1278 & $\begin{array}{l}\text { Essential for } \\
\text { vitro growth }\end{array}$ & $\begin{array}{l}\text { AAA_23 } \\
\text { AAA_27 }\end{array}$ & $\begin{array}{l}5-123 \\
471-858\end{array}$ & $\begin{array}{l}1.2 \times 10^{-7} \\
4.5 \times 10^{-10}\end{array}$ & $\begin{array}{l}\text { ATPases Associated with diverse } \\
\text { cellular Activities (AAA) }\end{array}$ \\
\hline
\end{tabular}


Table 6: List of pathogen-specific gene products and their description as in TubercuList database

\begin{tabular}{|c|c|c|c|c|}
\hline Sr.no. & Gene ID & $\begin{array}{l}\text { Protein } \\
\text { length }\end{array}$ & Functional category & Protein description \\
\hline 1. & Rv0078B & 68 & Conserved hypotheticals & Conserved protein of unknown function \\
\hline 2. & Rv0157A & 42 & Conserved hypotheticals & Conserved protein of unknown function \\
\hline 3. & Rv0397A & 82 & Conserved hypotheticals & Conserved protein of unknown function \\
\hline 4. & Rv0456B & 57 & $\begin{array}{l}\text { Virulence, detoxification, } \\
\text { adaptation }\end{array}$ & $\begin{array}{l}\text { Possible antitoxin mazE1. Function } \\
\text { unknown }\end{array}$ \\
\hline 5. & Rv1507A & 167 & Unknown & Hypothetical protein. Function unknown \\
\hline 6. & Rv2142A & 71 & $\begin{array}{l}\text { Virulence, detoxification, } \\
\text { adaptation }\end{array}$ & $\begin{array}{l}\text { Possible antitoxin parD2. } \\
\text { unknown }\end{array}$ \\
\hline 7. & Rv2237A & 78 & Conserved hypotheticals & Conserved protein of unknown function \\
\hline 8. & Rv2274A & 82 & $\begin{array}{l}\text { Virulence, detoxification, } \\
\text { adaptation }\end{array}$ & $\begin{array}{l}\text { Possible antitoxin mazE8. Function } \\
\text { unknown }\end{array}$ \\
\hline 9. & Rv2395A & 71 & Conserved hypotheticals & $\begin{array}{l}\text { Acid and phagosome regulated protein A. } \\
\text { Function unknown }\end{array}$ \\
\hline 10. & Rv2395B & 54 & Conserved hypotheticals & $\begin{array}{l}\text { Acid and phagosome regulated protein B. } \\
\text { Function unknown }\end{array}$ \\
\hline 11. & Rv2548A & 124 & Conserved hypotheticals & Conserved protein of unknown function \\
\hline 12. & Rv2645 & 143 & Unknown & Hypothetical protein. Function unknown \\
\hline 13. & Rv2658c & 120 & Insertion seqs and phages & $\begin{array}{l}\text { Possible prophage protein. Function } \\
\text { unknown }\end{array}$ \\
\hline 14. & Rv2862A & 82 & $\begin{array}{l}\text { Virulence, detoxification, } \\
\text { adaptation }\end{array}$ & $\begin{array}{l}\text { Possible antitoxin vapB23. Function } \\
\text { unknown }\end{array}$ \\
\hline 15. & Rv3032A & 129 & Conserved hypotheticals & Conserved protein of unknown function \\
\hline 16. & Rv3136A & 110 & Conserved hypotheticals & Conserved protein of unknown function \\
\hline 17. & Rv3190A & 69 & Conserved hypotheticals & Conserved protein of unknown function \\
\hline 18. & Rv3599c & 27 & Unknown & Hypothetical short protein. \\
\hline
\end{tabular}




\section{Legends to Illustrations:}

Figure-1: Workflow. The protocol used to analyse 4018 proteins in M. tuberculosis is outlined in this figure. The green coloured boxes correspond to categories of interest elaborated in the article.

Figure-2: Percentage residue coverage achieved in terms of domain assignments. A bar graph representation of percentage residue coverage achieved for proteins in context of domain assignments is depicted in this figure. The residue coverage achieved for 3566 proteins is divided into 10 bins (x-axis), and the number of proteins corresponding to each bin is provided (y-axis).

Figure-3: Overview on annotations. A pie representation of the overall percentage distribution of 4018 proteins in $M$. tuberculosis under each category identified. Five categories are illustrated: blue region $(89 \%)$ of the pie corresponds to proteins with recognisable structural and functional domains; red region (5\%) corresponds to proteins whose functions were identified by means of their evolutionary relationship to proteins of known function; orange region (2\%) corresponds to proteins whose folds were determined based on proteins of known structure; green region (3\%) corresponds to proteins identified to be specific to mycobacteria and light green region (1\%) corresponds to proteins specific to M. tuberculosis H37Rv.

Figure-4: Details of few of the new domain assignments. A) Predicted fold depicting 12-helix bundle adopted by Rv3630 (Region: 22-428) suggesting its possible role in multidrug transport. B) Secondary structural elements predicted in Rv0268c; highlighted regions constitute antitoxin domain. C) Crystal structure of toxin-antitoxin complex relBF from $M$. tuberculosis (PDB code: 3G5O) where the antitoxin component, predicted to be homologous to $R v 0268 c$, is shown in green and the toxin component is shown in cyan. Figure-4(a), Figure-4(c) and Figure-6 are prepared using PyMOL [76] (http://pymol.org).

Figure-5: Enhancing structural content of $M$. tuberculosis proteome. A pie-chart depicting percentage distribution of structural content with respect to Pfam domain families identified in $M$. tuberculosis. As illustrated in the figure, purple region (2\%) of the pie corresponds to 34 structurally uncharacterised domain families which could be related to structural families from SCOP.

Figure-6: De-DUFing DUFs. 6-bladed beta-propeller fold predicted to be acquired by members of DUF4185 family. Illustrated here is the predicted fold of $R v 1754 C$ (Region: 257-522; DUF4185 domain region: 230-560). 
Figure-7: Annotations for hypothetical proteins. A flow chart enumerating the annotations achieved for 955 of 1146 hypothetical proteins in M. tuberculosis. The hexagonal boxes denote the number of hypothetical proteins experimentally verified to be essential for pathogen [71-73].

Supplementary Figure-1: A bar graph representation of 15 most frequently occurring domain families in M. tuberculosis proteins. Black bar represents the number of occurrences of domains and gray bar corresponds to number of gene products housing the concerned domains. 


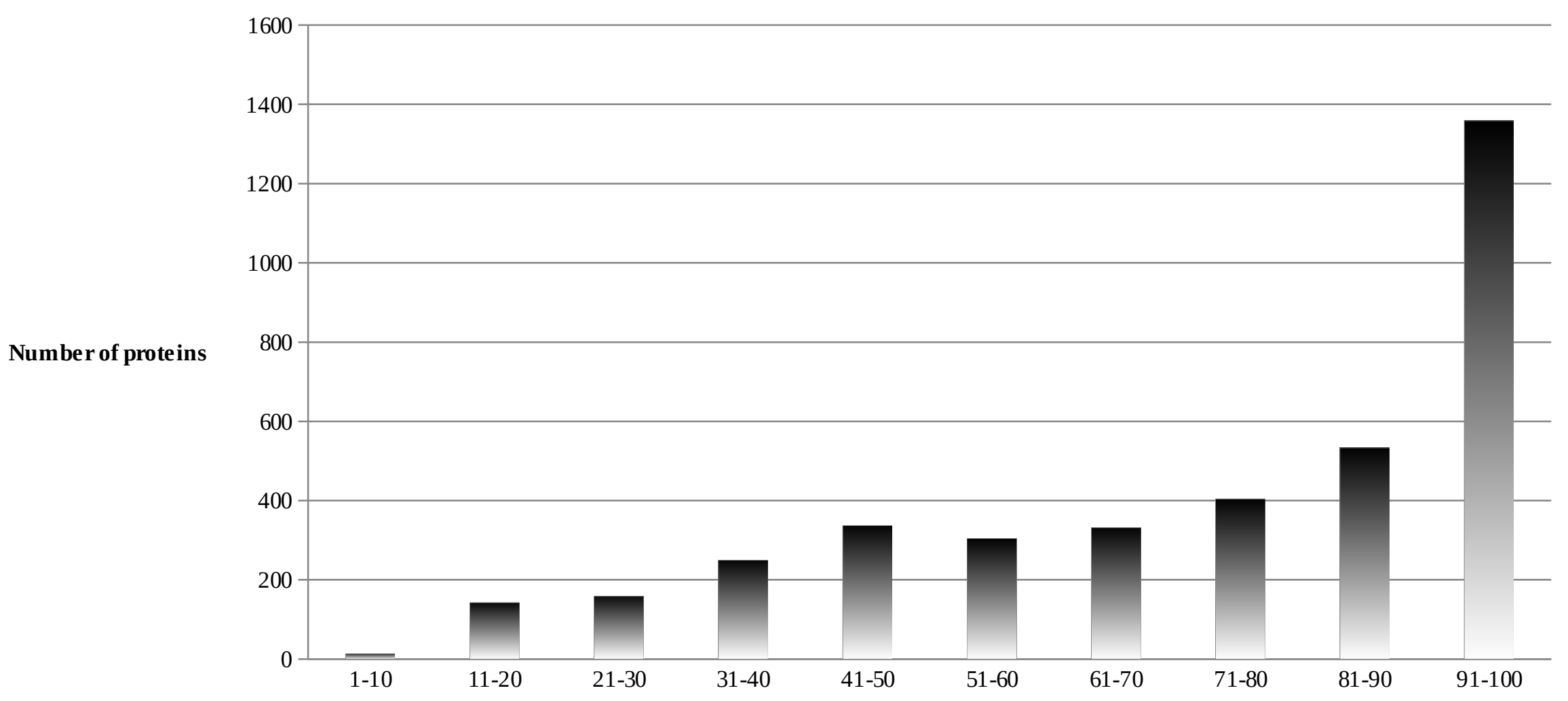

Range of percentage residue coverage for the proteins in terms of domains assigned 


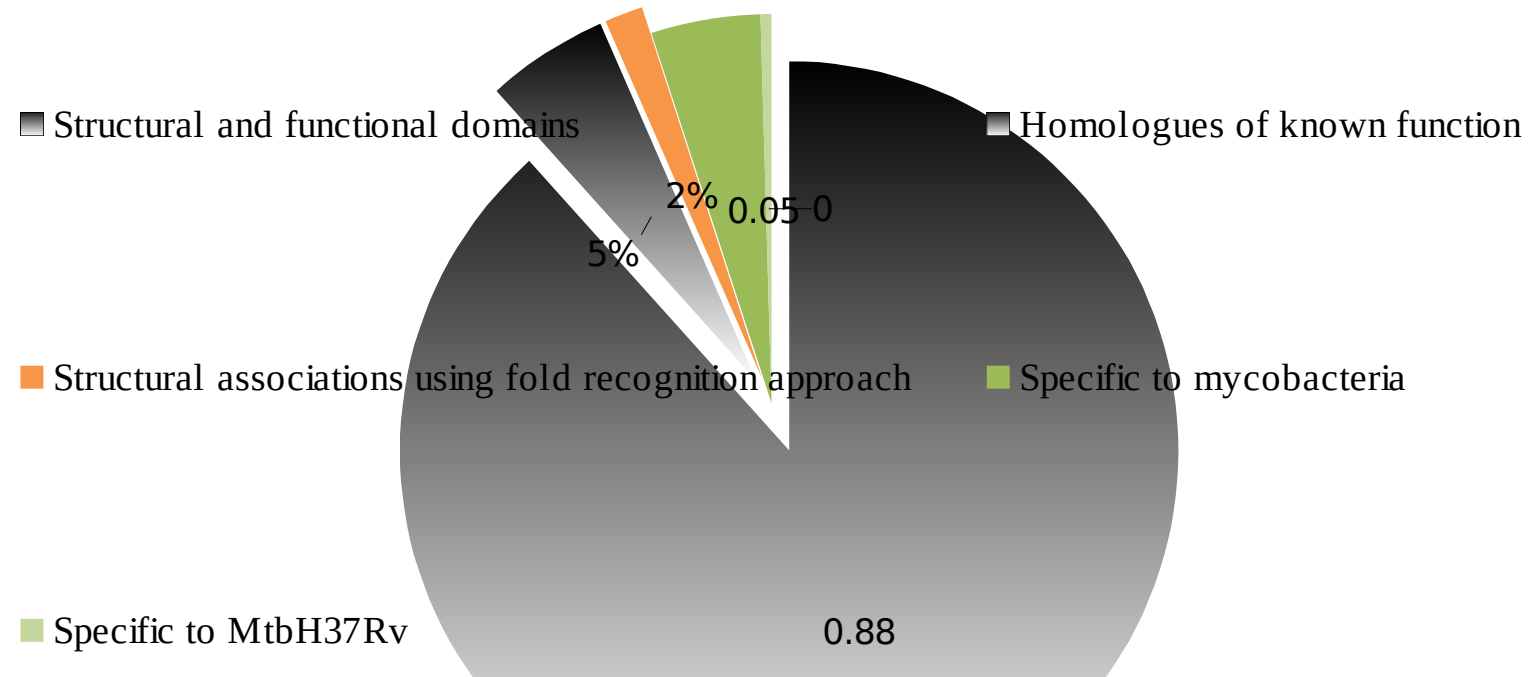


Domain families with known structural information

$5 \% 2 \%$

Domain families with no structural6øaformation

Domain families in Pfam clans associated with structural information

0.77

- Domain families with no structural information, associated with a known structural family 

Annotations for 955 of 1146

hypothetical proteins

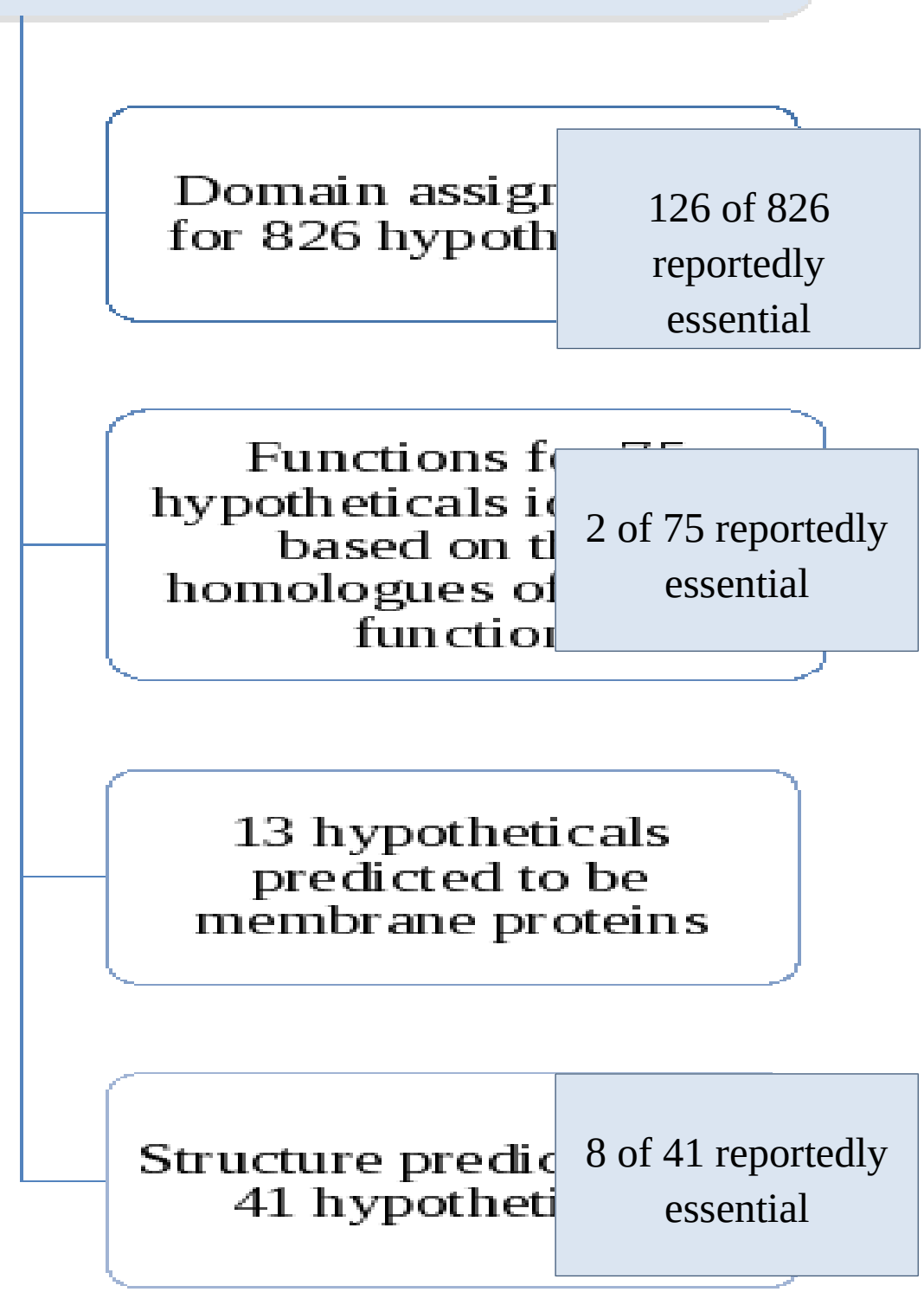




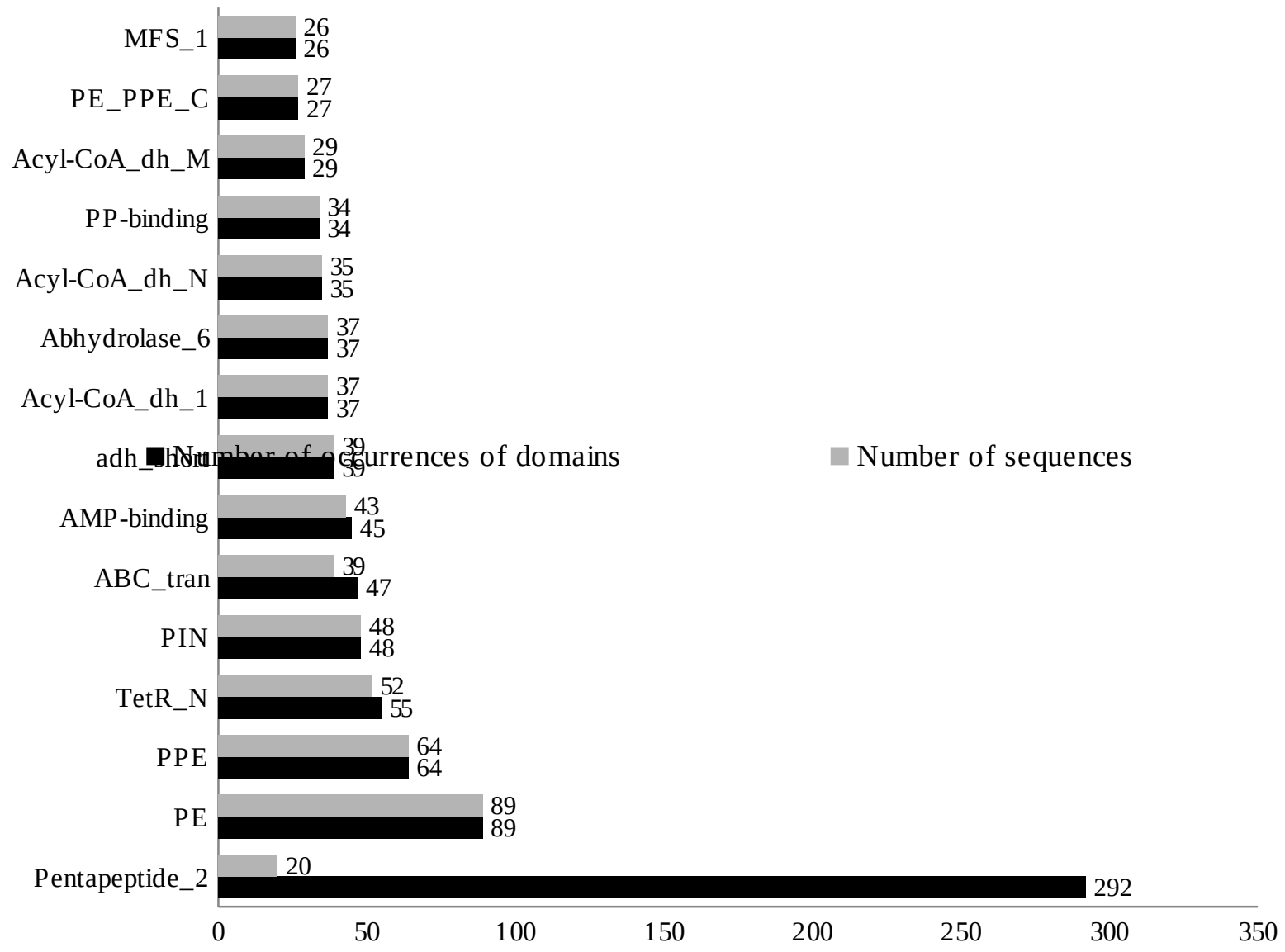

